\title{
CRYPTOCOLLEGE: HOW BLOCKCHAIN CAN REIMAGINE HIGHER EDUCATION
}

\section{J. David Judd*}

New Orleans Baptist Theological Seminary, Inxeption, 379 Oyster Point Blvd., South San Francisco, California 94080, USA

*Address all correspondence to: Address all correspondence to: J.D. Judd, New Orleans Baptist Theological Seminary, Inxeption, 379 Oyster Point Blvd., South San Francisco, CA 94080; Tel./Fax: (+1) 678-361-7744; E-mail: david.judd@inxeption.com.

\begin{abstract}
Blockchain is a relatively new technology that holds the promise to reimagine the future of higher education. The first commercial use of blockchain was born in the financial industry with the inception of Bitcoin, opening many new ways to conduct financial transactions. Blockchain now has the potential to disrupt higher education. More than the technology, the concept of an open and democratic system of trust can transform higher education in ways that will invite more participation while guaranteeing that all actors receive credit for their contribution. Three areas where disruption is occurring now are identity and digital credentials, content ownership and collaboration, and financing and funding. As blockchain becomes more mature and in wider use, institutions of higher education must consider the implications of this new technology in order to remain agile and relevant. Institutions should be aware of the potential dangers in ignoring, misunderstanding, or adopting too early this technology. Blockchain holds strong potential to deliver a new hybrid model for higher education that supports lifelong learning on a global scale.
\end{abstract}

KEY WORDS: blockchain, technology, credentials, funding, content ownership, community, collaboration, higher education, student debt, online education

\section{INTRODUCTION}

Every once in a while a new technology disrupts the status quo and allows us to connect with each other in new and different ways. The story of digital connectedness begins with the arrival of the internet, which gave the ability to send a message from one faceless computer administrator to another faceless computer administrator. At first, the messages were mundane and had to do with making sure the system was up, jobs were processed, and schedules for maintenance were broadcast. Then a strange thing happened. 
Operators began sending more personal messages to people they knew on the network. The messages connected administrators into a community of people with a shared interested in mainframe computers. Eventually, the administrators gave a name to this ability to send messages and called it e-mail. Miklacic (2015) posts a humorous clip on YouTube of Bryant Gumble and Katie Couric in 1994 on the Today Show trying to understand "What is the internet?" Some feel the same way in trying to explain blockchain today.

The pattern of technology disrupting the way people connect with others continued with the development of a computer program called a browser. Rather than people connecting through a green screen terminal, browsers could support colorful graphics that allow users to interact with other users easily through web pages. The World Wide Web was the term used to describe the interconnecting web pages created on the internet. Facebook introduced the ability to easily create a personal website to post pictures, status updates, and personal information. Now, the everyday person could connect with a community of like-minded people in a wide array of special interests. In the same manner, the mobile applications (apps) connected people in community through their smartphones with tools like Twitter and Instagram. New technology provides new ways for people to connect. Mougayar (2016) observes that blockchain applications may replace legacy web applications as well as "legacy businesses that cannot loosen their grips on heavy-handed centrally enforced trust functions."

\section{BLOCKCHAIN AS A TECHNOLOGY}

Today, blockchain is being thought of as the next generation of technology that allows people to connect to other people through their computers. Mougayar (2016) argues that "blockchain is not just any new technology (but that) it is a type of technology that challenges other existing software technologies, because it has the potential to replace or supplement existing practices." The significance of blockchain is that it breaks the past paradigm of having a central authority determine how people connect and what data they share. In the past, your e-mail provider or your cell phone carrier provided the public network that allowed you to send communications. With blockchain, computers connect directly to transmit encrypted messages to each owner. No central authority exists to broker the communication. Concerns about privacy, security, and sharing of personal information are why one would look to blockchain technology.

\subsection{What is Blockchain?}

Blockchain is like a digital ledger that's shared across a decentralized network of independent computers that are updated and maintained in a way that allows anyone to prove the record is complete and uncorrupted. Drescher (2017) states that the core 
problem that blockchain solves is to "achieve integrity and trust in a distributed system in the worst of all conditions." A blockchain maintains its integrity with a special algorithm embedded into a common piece of software run by all the computers in the network. The algorithm consistently steers the computers toward a shared consensus on what new data can be added to the ledger. Each computer updates its own version of the ledger independently but does so following a consensus algorithm. Once the new ledger entries are introduced, special cryptographic protections make it virtually impossible to change the entry.

Blockchain is the technology that operates digital currency systems like Bitcoin and Ethereum. The components of blockchain are a set of interrelated technologies: a distributed record of digital events, the distributed consensus method to agree whether a new block is legitimate and added to the record, automated smart contracts, and the data contained in each block.

A difference between blockchain and the internet in general is that the internet was built to connect people with information. The internet was built to move and store information, not necessarily to move and store data. To clarify, when one sends a document online, a copy of the file is sent. For example, one can forward a PowerPoint presentation in an e-mail, but the original presentation, the data, still remains with the sender. Only a copy of the file is sent. One can print a copy of the presentation, but again, the original file still remains in the possession of the author.

In contrast, a blockchain moves the actual data. A guiding principle of a blockchain is that no object of value can be duplicated. So, when a file is transferred on a blockchain, the original document is transferred. A second principle of a blockchain is that it permanently records transactions in a way that cannot be altered, erased, or hacked. Data on a blockchain can only be sequentially updated and therefore it keeps a permanent historical trail. In this way, a blockchain is like a database that is only ever updated and never overwritten.

\subsection{Blockchain in Digital Commerce}

To further explain blockchain, consider how money is used. When goods or services are procured, paper money is exchanged. An individual does not maintain the right to the money once it has been exchanged. It is illegal to print a copy of money. Once the money has been used, the ownership transfers to the new holder. Blockchain operates likewise. Since a blockchain creates a distributed record of digital events which cannot be changed, it is useful to record the transfer of ownership of original items of value such as digital music, property deeds, or academic records. 
To illustrate how blockchain is transforming the way data is moved, consider a typical Amazon purchase. One has to create a user account on Amazon and share personal information such as name, address, and credit card number. Amazon uses that information to offer items for sale. As one transacts commerce over time, Amazon will use personal information to offer more choices tailored to the consumer's past buying preferences. The more one uses the platform, the more Amazon knows about the buyer. However, the data about the transaction is not owned by the buyer. The data is shared with advertisers and those who would pay to have the buyer's information.

When one purchases an item, a credit card is used to broker the transaction. The bank issuing the card is the central authority facilitating the financial transaction. No money is exchanged directly and the bank takes its cut in transaction fees. Every purchase has an enormous amount of information about the buyer, the item, and transaction that is shared with third parties, often without express permission. If the transaction is facilitated on a blockchain, the buyer and seller are directly connected through their computers by a process of sharing secret permissions. The financial part of the transaction is conducted by offering something of value which is mutually agreed to by each party called a token.

The type of token is unique to each type of blockchain. Much like reward points in an airline program, the token has an agreed to value only between the parties in the transaction. Therefore, one sells an item for a certain number of tokens. The tokens are original and unique and cannot be duplicated, so the ownership is transferred from buyer to seller. The transfer of tokens is documented in a ledger that is shared by both parties. Neither party in the transaction can change the record, which becomes permanent on the blockchain. In this example no central bank brokers the transaction to take a percentage. No central store exists to mediate the order, so the information about what is bought and sold, to whom, and for what price remains private. No central authority has to give permission for the transaction to be completed.

A blockchain-enabled e-commerce platform has enormous potential to facilitate transactions between businesses. Unlike a consumer transaction, which has minimal continued interest beyond the acquisition of the item, a business transaction has implication for an extended relationship built on continued trust. The business may be procuring an item that requires a permanent record for maintenance and support. The bill of material for the item may be composed of thousands of serialized parts that need traceability and lot number control. The item may have an aftermarket for the product that extends beyond the initial buyer. A blockchain can store all of the product information in a way that is a permanent record of the manufactured part. Think of the blockchain as a CarFax for industrial products. 
Another benefit to a blockchain-enabled e-commerce transaction is no central bank exists to take a percentage of the transaction. Most businesses buy "on account" and not with a credit card. Few companies will have a credit card with the open-to-buy required for large capital expenditures. Financing and payments are contracted through purchase orders and invoices. A blockchain-enabled e-commerce platform facilitates buying and selling on the internet the way most companies have traditionally conducted business. Inxeption is an example of a business-to-business (B2B) e-commerce on blockchain.

The e-commerce example illustrates the benefits blockchain brings to disintermediated transactions requiring a permanent record of the account. Buyers and sellers can be confident that the information shared is required to conduct the transaction. The value of the transaction is negotiated between buyer and seller independent of outside influence. And the item procured exists in a permanent ledger which cannot be altered or hacked. Blockchain removes the unnecessary expense, inefficiency, and mutability to transactions. Higher education can leverage the e-commerce model to build models of trust and integrity when dealing with stakeholders like students and institutions.

\subsection{Distinctions in Blockchain}

Before considering the potential application of blockchain to areas of higher education, an important distinction defining types of blockchain should be discussed. Three types of blockchain exist. A public blockchain is one where membership is open to anyone and the network is defined as the connected computers participating in the blockchain. In a public blockchain no one entity controls the network and the blockchain is truly distributed. A private blockchain is more like a traditional network where a company owns the network and invites users to join the network. The problem with a private blockchain is it invites the middleman back into the transaction. A consortium is the third type of blockchain which seeks to maintain the autonomy of a distributed network and minimize the intrusiveness of a single controlling entity. A consortium is partly private and operates under the guidance of a leadership group. A consortium blockchain is a model well suited for higher education because it operates with governance similar to current accreditation organizations.

A second consideration of blockchain technology is the vast amount of energy and time required to maintain the system. Blocks are the unit of storage which contains the transaction information. Blocks not only contain the current transaction information but also all the information about the past transactions. When a new transaction needs to be recorded, a new block is created and linked to the previous block. The chaining of blocks through their historical records is where blockchain derives its name.

One of the reasons given for why blockchain is not a feasible technology for systems of higher education is because of the enormous amounts of time and energy needed to 
create a block for data. Blocks can be created two ways. One way is by using complex mathematical computations to encode the transaction information, which fits the new block uniquely on the chain like a piece of a puzzle. If the piece does not fit the puzzle, it is rejected. It is the job of independent computers called miners to figure out the way the new piece fits. The first computer to come up with the correct calculation is awarded compensation and their block is added to the chain. Using miners to create new blocks is resource intense and time consuming, therefore the method is called "proof of work." Bitcoin uses proof of work to create new blocks and rewards miners in bitcoin.

However, a second way to create a block of data that is quicker and less labor intense is through proof of stake and relies on a member voting process. In a proof of stake system, the members of the blockchain can validate the new block of data based on the amount of stake they hold in the system. Proof of stake is usually determined by evaluating how many tokens the members hold. Ethereum is the name of a blockchain that uses proof of stake to create new blocks. Higher education systems built on proof of stake would run more efficiently with greater control.

A blockchain can be used to store more than traded cryptocurrency transactions, and here is where blockchain gets really interesting. Ethereum, for example, makes use of a device called a smart contract to manage transactions according to a predetermined set of rules that are stated in a computer program. If conditions of the contract can be validated by the computer, then the transaction will occur automatically. A smart contract contains the prenegotiated terms that can validate and enforce the transaction. In general, if the terms of a standard contract can be defined in ways that a computer program could process the verification of performance, then a smart contract could be enforced on a blockchain. A self-executing contract provides the means to adopt the blockchain to applications seeking to transfer something of value based on performance of the parties to the transaction. If one complies with the terms of the contract, then something of value will be transferred. Smart contracts contain the ability to embed rules and policy into higher education workflow.

\section{PRACTICAL APPLICATIONS OF BLOCKCHAIN IN HIGHER EDUCATION}

Tapscott and Tapscott (2016) believe academia holds a critical role in the way blockchain is changing culture. Higher education is partnering with technology startups to create networks of universities interested in supporting education about blockchain and incubating new use cases. A blockchain sponsored by a consortium of higher education leaders with a vested interest in rewarding contributors based on shared values and common goals represents the best model for higher education. After discovering the technical aspects of blockchain and exploring the differences in types of blockchain, one 
can consider different applications of the technology to the issues facing higher education. Three areas in the realm of exploration seem ready for disruption by blockchain: credentials, content ownership, and finance.

\subsection{Identity Management and Credentials}

Identity management and credentialing have been at the intersection of higher education and business for a long time. Credentials are the currency of learning institutions. The only way employers can verify potential employees' credentials is by contacting individual educational institutions. Verifying every student's credentials is costly and time consuming. Employers are seeking a simpler and self-service way to verify an employment candidate's educational history. Currently, employers have to rely on the catastrophic consequences of providing false information to feel confident in the information provided on a resume.

Blockchain is used in a model of decentralized authentication to secure systems without the use of passwords. An application from HYPR identifies users' credentials safely on personal devices such as iPhones and Android phones using biometrics instead of passwords. Blockchain solutions eliminate centralized passwords by establishing the personal device as a holder of trust. Using decentralized user authentication, the person is in control of their identity and can determine what information is released to which organization. Likewise, IBM has an application to manage trusted identity through a decentralized, point-to-point exchange of information about people, organizations, or things. Cuomo (2018) challenges one to see a world when a person can control how much information one shares with a third-party. He sees blockchain as the path to self-sovereign identity.

Sharples and Domingue (2016) speculate that a blockchain could provide public evidence that a student identity received an award from an institutional identity. The University of Nicosia became the first university to award blockchain-based degrees. MIT offers a digital diploma verified on a blockchain. BADGR and Mozilla Open Badge are already being used to provide digital certifications to students in higher education institutions. Sharples and Domingue note that there are issues raised by trading educational reputation as a currency. They say, "One practical problem is how to create a conversion rate between reputation and money. What is the financial value of a novel idea or a dissertation? A fundamental ideological concern is that a system of trading reputation will further entrench the commodification of education - where students browse, buy, and consume educational products, with no empathy for scholarship or intellectual value."

Grech and Camilleri (2017) observe that the blockchain represents an opportunity for third parties, such as employers, to independently and privately verify that shared records are authentic and unadulterated. If employment and criminal records were stored in a 
blockchain, university human resource professionals could streamline the vetting process and move hiring processes forward more quickly. Credly, Acclaim, and Accredible are some companies providing digital credentials. Two technology initiatives that support issuing badges for certification are Blockcerts and OpenBlockchain. Applications built with these technologies issue and verify blockchain-based official records including academic credentials and professional licenses for doctors and lawyers. Other companies that have systems using self-identification and crowdsourcing to verify credentials are Indorse, Disiplina, Skill Safari, Echolink, and Skillchain.

\subsection{Content Ownership and Collaboration}

Chen et al. (2018) propose the use of blockchain in education to reduce or eliminate the threat of student and instructor data theft. For example, student records which are stored on blockchain cannot be modified. Students can attempt multiple courses and their academic history will be published on their public profile powered by blockchain technology. The ability to have a student profile that follows the student independent of the learning institution has implications for content ownership, transferability of credits, instructor recommendations, and promotion of lifelong learning. Intellectual assets can be easily maintained on a blockchain to track publications and citations. Bernstein Technologies allows companies to create a digital trail of records and intellectual property using blockchain technology providing proof of existence and ownership.

Filippova and Voshmgir (2018) argue that the publication and peer review process for academic research is stuck in an outdated preinternet era. The only place to publish is in academic journals, and the only place to defend the research is at academic conferences. The current norm is criticized for its slowness, reproducibility, and potential for tampering, misreporting data, and plagiarism. Filippova and Voshmgir believe that blockchain potentially creates a new platform for academic research built around the three pillars of academic research: collaboration, funding, and publishing. Blockchain offers transparency and efficiency, tamper-proof results, and an open and collaborative communication process. Blockchain can reward different researchers' contributions through tokenized incentives. The authors say a fair funding process would give the opportunity for any scientist, regardless of his or her career status, to receive proper funding for their research.

Blockchain provides the platform where content can be exchanged without the author losing ownership. Content can be exchanged as professors share their teaching material with their students. Adjunct professors can benefit from using course material created by instructional designers without the author losing control of the original material. Codevelopment of material can be fostered where teachers collaborate across disciplines 
to create classes. Finally, universities can be the catalyst for a global network of faculty, students, and corporate resources to develop collaborate learning.

Several blockchain companies have emerged for scientific publishing. Scienceroots is an example of a company investing in academic research on a blockchain. Scienceroot Journal offers tokenized currency to fund research and reward peer review. Every article is stored in an immutable archive. Pluto breaks down barriers in academia by opening scholarly communication to all researchers on a blockchain where no single individual or group has control of the system. Articles are recorded on a public blockchain and researchers are compensated by securing their academic reputation. DEIP is a decentralized research platform that is sustained by issuing tokens. DEIP maintains a database of science grants which are given based on consensus by the active community. Database company MaterialsZone received a million dollars in funding to develop a community-driven platform to allow researchers to share and sell their data. MaterialsZone's focus is building an open science economy. Artifacts aims to publish scientific papers with all the research data usually left out of articles with the opportunity to reproduce results.

\subsection{Finance and Funding}

Craig (2018) predicts blockchain will transform the way higher education sees finance and funding, especially in the area of student debt. Because blockchain represents a transparent, verifiable register of transaction data operating on a decentralized platform, it is fraud resistant. Student funds can be received using blockchain-based smart contracts to provide all the funding for education up front. The smart contract will validate performance against a degree program so that funds are incrementally released to pay student tuition as the student successfully completes classes. Educational institutions will issue credentials after the blockchain releases the funds.

Blockchain opens the possibility of using a type of crowdfunding to solicit resources for tuition. Moru (2018) explores the idea of an income share agreement (ISA) as a way to finance a student's education based on a contract to repay the lender a percentage of the student's future income. For example, the student can payback $10 \%$ of their income for 10 years to an ISA company. The difference between an ISA and a loan is that the student does not begin to pay back the money until they get a job. Goal Structured Solutions and Vemo Education are companies that administrate innovative solutions in education finance.

\section{OPPORTUNITIES AND WARNINGS}

The traditional classroom context for education is a hurdle for adults seeking to extend their learning path into their career. The opportunity exists for blockchain to deliver on the 
promise of lifelong learning first offered by online education. Bowen (2013) speculates that using new technologies can address the problems of traditional education. Internet-based university education promotes a hybrid model to serve more adults with education, which often happens in environments outside the traditional classroom. Corporations deliver specialized training, skill assessment, and professional certification. Often corporate training and certification is on par with university standards. In addition, vocational training and industry credentials are recognized as attainment of expertise in a subject.

Mikroyannidis and Bachler (2018) argue for a blockchain that offers a learner-centered approach for lifelong learning where educational content and training services combine to shape a career trajectory. A decentralized platform that recognizes the validity of corporate training alongside the traditional approach to education would provide a platform for learning bodies to collaborate and deliver a tailored learning path supporting a lifetime career. Blockchain can support a hybrid model that supplements traditional higher education with verified coursework from outside institutions. Students can customize a program to create new models of learning. OpenLearn University and FutureLearn are two systems that support hybrid education on blockchain.

The inherit virtue of the blockchain also contains a seed of caution. Casey and Vigna (2018) believe blockchain is capable of supplanting the current outdated model of trust management upon which societies and economies have functioned in the past. They see blockchain as "a social technology, a new blueprint for how to govern communities." Because the blockchain is democratic, there is no one entity that owns it. The move to independence is contrary to the way centralized autocratic educational institutions work today. While some schools are influenced by meritocracy, it will take a consortium of leadership to adopt a blockchain with full participation of all institutions.

\section{REFERENCES}

Bowen, W.G. (2013), Higher Education in the Digital Age, New Jersey: Princeton University Press, pp. 43-44.

Casey, J. and Vigna, P. (2018), The Truth Machine: The Blockchain and the Future of Everything, New York: St. Martin's Press, p. 14.

Chen, G., Xu, B., Lu, M., and Chen, N.-S. (2018), Exploring Blockchain Technology and Its Potential Applications for Education, Smart Learn. Environ., 5:1, Retrieved September 23, 2018, from https://doi.org/10.1186/s40561-017-0050-x.

Craig, R. (2018), How Blockchain Can Truly Revolutionize Higher Education, Retrieved September 1, 2018, from https://www.forbes.com/sites/ryancraig/2018/02/22/howblockchain-can-truly-revolutionize-higher-education/\#30801be126b7. 
Cuomo, J. (2018), IBM Blockchain Whitepaper: Technical Overview, Retrieved November 16, 2018, from https://www-01.ibm.com/common/ssi/cgi-bin/ssialias?htmlfid= KUW12555USEN.

Drescher, D. (2017), Blockchain Basics: A Non-Technical Introduction in 25 Steps, Germany: Frankfurt and Main, p. 31.

Filippova, E. and Voshmgir, S. (2018), Blockchain Solutions for Scientific Publishing, Retrieved September 1, 2018, from https://medium.com/crypto3conomics/blockchainsolutions-for-scientific-publishing-ef4b4e79ae2.

Grech, A. and Camilleri, A.F. (2017), Blockchain in Education, A. Inamorato dos Santos, Ed., Luxembourg: Publications Office of the European Union, Retrieved September 1, 2018, from http://publications.jrc.ec.europa.eu/repository/bitstream/JRC108255/jrc108255_ blockchain_in_education\%281\%29.pdf.

Miklacic, C. (2015), 1994: Today Show: What is the Internet Anyway? Retrieved September 1, 2018, from https://www.youtube.com/watch?v=UIJku_CSyNg.

Mikroyannidis, A. and Bachler, M. (2018), A Learner-Centered Apporach for Lifelong Learning Powered by the Blockchain, Waynesville, NC: Association for the Advancement of Computing in Education (AACE), Retrieved September 1, 2018, from https:// www.learntechlib.org/primary/p/184356/.

Moru, N.R. (2018), The Real Solution to Student Debt Crisis: Blockchain-Based Income Share Agreements, Retrieved September 1, 2018, from https://en.decentral.news/ blockchain-based-income-share-agreements/.

Mougayar, W. (2016), The Business Blockchain: Promise, Practice, and Application of the Next Internet Technology, New Jersey: John Wiley and Sons, pp. XIX, 10.

Sharples, M. and Domingue, J. (2016), The Blockchain and Kudos: A Distributed System for Educational Record, Reputation and Reward, in K. Verbert, M. Sharples, and T. Klobučar, Eds., Adaptive and Adaptable Learning, EC-TEL 2016, Lecture Notes in Computer Science, Cham, Switzerland: Springer, vol. 9891.

Tapscott, D. and Tapscott, A. (2016), Blockchain Revolution: How the Technology behind Bitcoin Is Changing Money, Business, and the World, New York: Penguin Random House, pp. 246-249. 